\title{
Tincani, Persio. Identità e meraviglia. Cinque scritti brevi di diritto, politica e letteratura. Milano: l’Ornitorinco edizioni, 2020
}

Francisco Javier Ansuátegui Roig

Universidad Carlos III de Madrid

Fecha de recepción 23/11/2021 I De publicación: 23/12/2021

Cuando uno se encuentra con un libro erudito, que rezuma curiosidad intelectual, y que propone una reflexión sobre episodios extraídos desde la literatura medieval hasta la literatura sobre robótica, la tarea de identificar elementos a través de los cuales se pueda articular una argumentación no parece fácil. En efecto, en el libro de Persio Tincani nos encontramos capítulos centrados en Beowulf, la Comedia, Don Quijote, Alicia en el país de las maravillas, o Preludio a la Fundación). Estamos frente a un libro vivo, con una dinámica interna que comienza con capítulos en los que hay un discurso más centrado en los textos de referencia y que termina con capítulos en los que los textos de referencia no constituyen tanto el centro de atención, sino que parecen una excusa o invitación para abordar otras cuestiones.

Antes de centrarme en alguno de los temas el libro, me gustaría hacer una reflexión general sobre lo fructífero del análisis de la relación entre el Derecho y la literatura; relación que puede ser entendida en el marco de la más amplia relación entre el Derecho y la cultura.

Entre Derecho y cultura existe una relación bidireccional. El Derecho es un elemento básico de una determinada cultura, implica un modelo de organización y es expresión de una autocomprensión del grupo humano. En este sentido, una determinada cultura no se entiende sin reconocer el papel del Derecho. Al mismo tiempo, la cultura condiciona el Derecho. Los rasgos culturales básicos de un grupo humano tienen una vocación de reflejo en el Derecho. E influyen en su funcionamiento. Esto lo comprobamos al estudiar las causas por las que una norma o un sistema jurídico puede ser ineficaz y fracasar como propuesta normativa. Entre ellas nos encontramos con la contradicción entre el sentido de las normas y los aspectos culturales básicos del grupo. Así, la relación entre el Derecho y la cultura es un elemento básico de la relación entre del Derecho y la sociedad, como bien saben los sociólogos del Derecho. 
No descubro nada si recuerdo que la literatura es una de las expresiones culturales más relevantes. Su relación privilegiada (y que la diferencia de la relación que se establece entre el Derecho y otras manifestaciones culturales) con el Derecho se puede analizar desde diferentes puntos de vista. El Derecho y la literatura comparten un medio: el lenguaje escrito. Sería interesante preguntarse si esta es la razón por la que otras expresiones culturales importantes, como la escultura o la música no han sido analizadas tan en profundidad en sus relaciones con el Derecho y la política (sobre esto hay excepciones, como las que tienen que ver con las bellas páginas que, por ejemplo, Quentin Skinner ${ }^{1}$ y Manuel García Pelayo ${ }^{2}$ han escrito en torno a la Alegoría del Buen Gobierno de Lorenzetti del Palazzo Pubblico de Siena). Con la excepción del fructífero análisis de la relación entre el Derecho y el cine $\mathrm{e}^{3}$-que hoy se ha consolidado como una estrategia pedagógica en algunas facultades de Derecho- que yo sepa, no hay un movimiento Derecho y pintura o Derecho y escultura, tan desarrollado como Derecho y literatura. La razón no puede tener que ver con que el Derecho y la literatura sean ámbitos en los que la interpretación es necesaria. Es una característica compartida también, por ejemplo, con la música, como nos recordó Jerome Frank ${ }^{4}$ y más recientemente Gustavo Zagrebelsky en su conversación con Mario Brunello ${ }^{5}$. Posiblemente, como he señalado, el hecho de compartir el lenguaje - el canal de expresión- esté en la base de la atención que prestamos a las relaciones entre el Derecho y la literatura.

¿Cuál es la utilidad del análisis de estas relaciones? ¿Por qué puede ser interesante para un jurista el análisis de las obras literarias? Derecho y literatura comparten espacio, el mundo de la vida. En este sentido, los problemas que se plantea el Derecho se relatan en la literatura, aunque no todas las dimensiones de la vida humana son de fácil localización y acomodo en el Derecho. Por otra parte, podríamos pensar, la frialdad del Derecho contrasta con la calidez de la literatura. La vocación de cumplimiento, de eficacia, que caracteriza el mensaje jurídico no existe en el mensaje literario. El lector, frente al mensaje literario, encuentra una libertad que no tiene frente al mensaje jurídico. Las

\footnotetext{
${ }^{1}$ Vid. Quentin SKINNER, El artista y la filosofía política. El Buen Gobierno de Ambrogio Lorenzetti, trad. de P. Aguado y E. García, Trotta, Madrid, 2009.

2 Vid. Manuel GARCIA-PELAYO, "El buen y el mal gobierno", en ID., Del mito y de la razón en el pensamiento político, Revista de Occidente, Madrid, 1968, pp. 319-337.

${ }^{3}$ Estoy pensando en la colección "Cine y Derecho" de la editorial Tirant lo Blanch.

${ }^{4}$ Vid. Jerome FRANK, “Words and Music: Some Remarks on Statutory Interpretation”, Columbia Law Review, 47(8), 1947, pp. 12591278. También, Andrea PORCIELLO, "Diritto e musica: armonia o dissonanza?, Materiales de Filosofía del Derecho, Universidad Carlos III de Madrid, 2018/5

(https://e-archivo.uc3m.es/handle/10016/27416\#preview)

${ }^{5}$ Vid. M. BRUNELLO, G. ZAGREBELSKY, Interpretare. Dialogo tra un musicista e un giurista, Il Mulino, Bologna, 2016.
} 
sensibilidades, las emociones, tienen un lugar en el mensaje literario que no existe en el jurídico. Podríamos considerar que la perspectiva de lo humano que nos ofrece la literatura es más amplia que la que nos ofrece el Derecho. La literatura ofrece un discurso sobre el sentido de las circunstancias que acompañan los asuntos humanos mucho más amplio que el que nos puede ofrecer el Derecho en su voluntad regulatoria. Esto es cierto, más allá del hecho de que la literatura carece de los límites con los que se encuentra, o se debería encontrar, el Derecho. No hay nada humano que sea ajeno a la literatura, que le esté prohibido. Por el contrario, hay muchos y relevantes ámbitos de lo humano en los que el Derecho no debería intervenir, de acuerdo con la mejor propuesta liberal.

En todo caso, y puestos a identificar las utilidades y ventajas de esta relación entre Derecho y literatura, podemos pensar en que la literatura, como elemento básico de la cultura, fortalece y enriquece la capacidad del jurista a la hora de enfrentarse con los problemas jurídicos. El jurista debería tener una sensibilidad que va más allá del conocimiento del Derecho, una sensibilidad que le permita comprender lo humano, sus problemas: esos son los problemas sobre los que va a trabajar, ese es su ámbito. En realidad, en pocas palabras, estoy pensando en algo que puede parecer tan banal, pero que en ningún caso lo es, como la diferencia entre el jurista culto y el jurista inculto. Aunque, pensándolo bien, no es tan banal pensar, con Nuccio Ordine, en el peso de lo aparentemente inútil -la formación humanista- en la educación del ciudadano (y del jurista en particular) ${ }^{6}$. Y no lo es, entre otras cosas, porque el jurista inculto va a tener muchas posibilidades de convertirse en ese jurista meramente técnico, en ese leguleyo, sin ninguna capacidad crítica, que tan cómodo es para el poder político. Una de las cosas que debe preocupar a la filosofía del Derecho es su responsabilidad en la formación de un determinado modelo de jurista. Por otra parte, la literatura ofrece un panorama en el que el jurista -y aquí el filósofo del Derecho posiblemente tenga una posición privilegiada- puede reconocer problemas, conceptos, realidades, que son las que le preocupan, pero sin los corsés del estricto lenguaje jurídico. En este sentido, la literatura se puede analizar en clave jurídica. Aquí el Derecho se convertiría en un criterio o perspectiva de interpretación de la literatura.

Pues bien, va a ser precisamente esta posibilidad la que voy a aprovechar en esta ocasión. Porque los cinco capítulos que componen el libro de Persio Tincani son una invitación a la reflexión ya que en ellos podemos identificar cuestiones (mostradas desde diferentes perspectivas) que, desde el punto de vista

${ }^{6}$ Vid. Nuccio ORDINE, La utilidad de lo inútil. Un manifiesto, trad. de J. Bayod Brau, Acantilado, Madrid, 2013. 
filosófico, e iusfilosófico en particular, son relevantes. Por supuesto, voy a tener que hacer un esfuerzo de selección en la identificación de las mismas, con el evidente riesgo de elaborar un discurso algo desequilibrado en relación con la atención que merecen los distintos capítulos. No es fácil abordar todos los matices y sugerencias del libro en su amplia extensión.

Uno de los temas, relevantes desde el punto de vista político, jurídico y moral, con los que nos encontramos en el libro, y más en particular en el primer capítulo, es el del mal. Pero el mal, personificado en la figura de Grendel, no es un mal sustantivo, sino aquel que se identifica con la actuación sin sometimiento alguno a reglas. En este sentido, Grendel es la negación del Derecho, es la arbitrariedad. Si la actuación de acuerdo con reglas es expresión de un cierto avance civilizatorio, Grendel es la negación de la civilización. No indemniza, no compensa los daños, no asume límites. Es un fuori norma que no reconoce la posibilidad de limitar la venganza. Es la negación de la idea misma de orden social que incluye mecanismos de gestión de la violencia. En la descripción del comportamiento de Grendel hay una invitación a la reflexión sobre el valor de las reglas y, por extensión, sobre el valor del Derecho.

El carácter desvinculado de Grendel provoca el que sea identificado por todos como el enemigo. La magnitud del peligro que supone, provoca al mismo tiempo la autocomprensión de aquellos que sufren el peligro de Grendel como una comunidad. Tiene un efecto aglutinador para aquellos que lo sufren. Por eso Persio Tincani lo presenta como el enemigo público, de todos, y además interno. Por cierto, el carácter interno, nos permite además reflexionar sobre una de las dimensiones del poder de Grendel, su capacidad de ocupar el espacio. Su poder no está vinculado a un territorio. Aunque viva lejos puede llegar al castillo. Esta es una dimensión relevante de su poder, que escapa a los límites espaciales. Límites que son importantes en la comprensión moderna del Derecho, vinculado a la noción de territorio. En realidad, la lejanía de Grendel no se puede explicar en términos geográficos. Es una lejanía conceptual.

Estamos frente a un enemigo del orden, pero no de un orden concreto y coyuntural, sino de la misma idea de orden. Y, por tanto, podríamos pensar, de cualquier cosa que se pueda aproximar al Derecho. En el análisis que un jurista podría hacer de esta situación, aparecería tarde o temprano el recurso a las normas, la confianza en las reglas. Esa confianza la hemos extendido incluso a la regulación de la que podría ser considerada la expresión máxima de la violencia, la guerra. Pero lo inquietante, y lo trágico de la situación, es que parece que la magnitud de la anormalidad que se describe muestre la insuficiencia de las reglas. Es tal la magnitud de la anormalidad, nos encontramos tan lejos de cualquier orden 
constituido, que las reglas no sirven como respuesta. No es un desorden normal, uno de aquellos conflictos que pueden ser regulados jurídicamente.

Persio Tincani nos invita a pensar en los límites del Derecho. Por cierto, idea ésta de límite vinculada a la de identidad, ya que la idea de identidad implica establecer contornos diferenciados y diferenciadores: el límite como lo que no se puede hacer, la limitación; y el límite como lo que diferencia. Pues bien, funcionar de acuerdo con un sistema de reglas implica a asumir una limitación. Pero parece que frente al mal ilimitado las reglas no sirven. Ese es aquí el problema.

Estamos frente a una situación que debería inquietar al jurista. Grendel sólo puede ser vencido si no se recurre a las reglas. A partir de ahí, surge la pregunta: ¿el Derecho no sirve, o no sirve siempre, frente al mal radical? ¿Luchar contra el mal radical implica necesariamente convertirse en un fuori norma? Parece que eso es lo que le ocurre a Beowulf.

Pero, posiblemente, más que hablar de límites del Derecho, deberíamos hablar de limitaciones del Derecho, pensando en aquellas circunstancias en las que el Derecho muestra sus insuficiencias, es incapaz de hacer aquello para lo que está pensado, resolver el conflicto de acuerdo con reglas que, sin son justas, mucho mejor. Lo trágico de la situación es que tenemos que llegar a la conclusión de que tanto Grendel como Boewulf, tanto el monstruo como el guerrero, tanto el anticristo como aquel que quiere terminar con el anticristo, están más allá del Derecho. Es tal la potencia, la magnitud, del anticristo, que la reacción contra el mismo implica escapar de la civilización, huir del criterio civilizatorio que significan las normas, las reglas. Para el Derecho, el bien inicial que podría significar la lucha contra Grendel, se convierte en un mal desde el momento en que presenta el mismo carácter ilimitado que el poder del monstruo. Al fin y al cabo, el Derecho no sólo nos invita a pensar en fines, sino que nos exige pensar en medios. Principalmente, podríamos decir, el Derecho es una cuestión de medios, aunque no exclusivamente; exige de nosotros una actuación de acuerdo con las reglas. Y si a partir de aquí queremos extraer consecuencias referidas a la moralidad de la misma existencia del Derecho, consecuencias que afectan a la relación entre el Derecho y la moral, nos sumergimos en uno de los aspectos básicos de la teoría del Derecho. Aunque esta es una cuestión en la que no nos podemos detener en este momento.

En límites también podemos pensar cuando leemos la historia de Ulises, que nos relata Dante (más allá de la posibilidad - en la que tampoco me centro ahora - de reflexionar sobre la mentira, ya que Ulises se presenta como un gran mentiroso en el episodio del caballo de Troya; ello nos conduciría a pensar en la 
relación entre verdad/mentira y política, tan relevante en la historia de la filosofía política y de la filosofía moral y tan urgente en tiempos de posverdad como los nuestros). Pero aquí, en la historia de Ulises, los límites con los que nos encontramos son dos, aunque relacionados: por una parte, el límite geográfico que no puede traspasar; por otra, la norma establecida por los dioses y que señala que no debe traspasar ese límite. Ulises no es Grendel, y tampoco Beowulf; a él sí le afectan los límites. Por tanto, la posible reflexión es muy diferente.

El problema de Ulises es el de la violación de una norma divina, la que establece la prohibición de traspasar el límite establecido por Hércules. La invitación a pensar en el eterno problema del Derecho natural me parece evidente. Si bien el enfoque puede ser diferente de aquel que identifica el Derecho natural como un criterio de validez de las normas dictadas por los hombres. Aquí, el Derecho natural funciona como un elemento de distinción -la distinción implica recurrir a la idea de límite- entre lo humano y lo divino, entre los ámbitos en los que se puede desenvolver y decidir el ser humano, y aquellos otros que están reservados a la divinidad. Traspasar los confines de lo reservado al individuo, invadir el espacio de la divinidad, es expresión de la tentación de convertirse en Dios, lo cual supone alterar el orden y la armonía del Derecho natural. Esta dimensión limitativa del Derecho natural -que tiene consecuencias tanto conservadoras como revolucionarias- es una constante a lo largo de su historia y llega a nuestros días -salvando todas las distancias- en forma de constitucionalismo. Pero esta es otra cuestión.

Los límites que ha impuesto el Derecho natural son límites a la decisión, a la acción. Pero aquí, en la historia de Ulises, el límite lo es ciertamente al conocimiento. En realidad, este límite al conocimiento, en cuanto tal, también lo es al poder, puesto que el conocimiento es poder. Limitar el conocimiento conduce a la limitación de la capacidad de decidir. Estamos frente al problema de la existencia del secreto como ámbito y estrategia del poder. El que conoce el secreto domina, tiene el poder. Por eso Persio Tincani llama la atención sobre el componente esotérico de toda religión. Pero, ciertamente, podríamos añadir que no sólo de toda religión, desde el momento en que el recurso a los arcana imperii trasciende en mucho al discurso religioso. Secretos, arcana, cuyo carácter escondido es al fin y al cabo un recurso del poder a la hora de establecer distancias con la comunidad y de asegurar la inmunidad de las decisiones que se adoptan de acuerdo con lo que sólo unos pocos conocen.

Actuar dentro de los límites es expresión de lo racional, de lo cuerdo, de lo adecuado, de lo normal. Por eso, la voluntad de traspasar los límites es expresión de la locura de Ulises. La idea de locura reconduce 
a la de exceso, a la de desmesura. El objeto del exceso puede ser diferente (pasión, confianza, temeridad, tristeza, alegría...), pero lo importante aquí es que el exceso implica desmesura, algo que no puede ser medido, y que por lo tanto escapa a cualquier discurso sobre el límite. Volviendo a una reflexión anterior, en este punto la regla que limita, que protege frente a la desmesura, no vale. Por eso lo contrario del exceso es la prudencia. Prudencia que implicaría, entre otras cosas, asumir las ventajas de actuar y decidir de acuerdo con reglas. Algo que tiene que ver con la iuris-prudencia. Las reglas, las normas, asumir el ius como criterio de acción, protege frente al exceso, frente a la desmesura, frente a la locura; locura que, en el caso de Ulises, va más allá de su voluntad de saltar unas leyes del universo, ya que en realidad esas leyes no pueden ser violadas, a diferencia de lo que ocurre con otras normas, posiblemente con la mayoría de las normas que afectan a los asuntos humanos. Unas leyes del universo imposibles, no tanto en cuanto a su cumplimiento como a su incumplimiento. Muy diferentes, por tanto, respecto a las leyes humanas, a las normas jurídicas, que han de ser posibles tanto en su cumplimiento como en su incumplimiento, ya que si el incumplimiento no es posible (y por tanto su cumplimiento es necesario), las leyes mismas pierden su razón de ser (algo que nos recordó Kelsen al explicar las diferencias entre las leyes de la naturaleza y las leyes humanas). De modo que a la locura de su voluntad hay que añadir la locura de su ignorancia, del desconocimiento de lo que es materialmente imposible. ¡Pobre Ulises, ignora que, en realidad, no puede hacer aquello que quiere hacer! Su esfuerzo va a ser inútil.

Al inicio del libro, Persio Tincani señala que se centra en el problema de la identidad. Yo he enfocado la reflexión asumiendo como referencia la idea de límite, lo cual es otra forma de pensar en la identidad. Ya que hablar de la identidad implica poder señalar lo no idéntico, establecer límites y distinciones entre ambos. Conviene recordar esto ya que cuando Persio Tincani nos habla de Don Quijote y de Alicia el problema de la identidad pasa a primer plano, preparando el terreno al problema que se planteará a raíz de la relación entre Hari Seldon e Dors Venabili. Pero vayamos por partes.

Tanto en el caso de Don Quijote como en el caso de Alicia nos preguntamos ¿quién es Don Quijote?, ¿quién es Alicia? Me parece que el sentido de las preguntas en cada caso es diferente.

Por una parte, en Don Quijote, nos encontramos frente a una tensión narrativa que obliga a diferenciar, casi constantemente, en unas ocasiones con más intensidad que en otras, entre el escritor y el protagonista de la historia. Esa no es la única dualidad con la que nos encontramos. Toda la obra está recorrida por una dualidad entre lo real y o ideal, que se manifiesta de manera muy gráfica en episodios como los de los molinos de viento en el capítulo VII de la primera parte. La confusión entre fantasía y experiencia 
constituye la clave de interpretación de las peripecias de Don Quijote, y al mismo tiempo la fuerza que le impulsa. Pero lo que quiero subrayar aquí no es tanto lo referido a esta dualidad sino otras distinciones como la que tiene que ver con aquella otra entre la justicia humana y la justicia natural, con la distinción entre lo que es adecuado para el Derecho y lo que lo es para la moral, con la diferencia entre el sentido objetivo y subjetivo de la justicia. La estrategia de Cervantes es la que consiste en presentar una realidad que nos invita a pensar en una alternativa: ¿y si la justicia no se identificara con la voluntad del rey, a diferencia de lo que señala Sancho? De la misma manera que nos invita a reflexionar -en el episodio de los condenados a galeras - también sobre la humanización del Derecho penal y procesal, adelantándose al discurso de la Ilustración.

En todo caso, -permítaseme añadir- la reflexión sobre la justicia que nos presenta Persio Tincani puede completarse con la alusión a los consejos que Don Quijote ofrece a Sancho, cuando va a ser nombrado gobernador de la ínsula de Barataria, de acuerdo con el relato del capítulo XLII de la segunda parte. Allí Don Quijote presenta en realidad el conjunto de las virtudes que ha de tener el gobernante que va a impartir justicia: humildad, compasión, voluntad de descubrir la verdad, moderación de la rigidez de la ley, misericordia, alejamiento de las pasiones, piedad y clemencia. En definitiva, el prolegómeno de una deontología de la función judicial.

En el caso de Alicia, creo que la cuestión de la identidad puede ser planteada a la luz de la reflexión sobre la naturaleza institucional del Derecho. El Derecho construye modelos de personalidad. La misma idea de persona es una construcción jurídica. Es precisamente en el marco de las reglas en donde adquirimos una determinada personalidad, una identidad, y en donde -además- podemos actuar en los marcos creados por las mismas reglas. Es ese marco de reglas el que atribuye sentido a nuestras actuaciones, pero no sólo. Porque actuar de acuerdo con las reglas es también una estrategia de integración. Participar del sentido compartido del juego de cartas, en este caso, genera comunidad, genera inclusión. Y lo contrario a la inclusión es la exclusión. La participación en los mecanismos del Derecho, en un juego o en un proceso, implica pertenencia; pertenencia que es un elemento definidor de la subjetividad. Pero no es la actividad en sí la que genera esa subjetividad, sino la existencia de reglas racionales -que dan sentido a esa actividad-, sin las cuales tenemos dificultades para identificar esa actividad en sí misma. Sin reglas no hay ni juego ni proceso: más bien nos encontramos frente a una situación arbitraria en la que tenemos dificultades para interpretarla en términos institucionales, y más concretamente jurídicos. El problema en el que nos invita a pensar Persio Tincani es el de la vinculación entre las reglas y una cierta 
racionalidad: ¿podemos encontrar en las reglas que no responden a ningún modelo de racionalidad esa capacidad de generar integración y comunidad?

Pero, posiblemente, la culminación de la reflexión sobre el problema de la identidad se encuentra en el último capítulo del libro, en donde la cuestión de la esencia y del sentido de lo humano recibe toda la atención. Es, aunque no sólo, aquí en donde nos encontramos con problemas de espesor fílosófico, porque en realidad la pregunta de fondo es la que se refiere al concepto de vida y a la distinción entre vida y no vida; distinción, en este caso, diferente a aquella otra entre vida y muerte.

Se acaba de señalar que el Derecho nos hace personas, nos atribuye una personalidad, pero ahora Persio Tincani nos invita a reflexionar sobre los límites de lo humano. A lo largo de la historia hemos identificado la vida, y por derivación lo humano, a partir de elementos orgánicos, carbónicos. Pero, como ha recordado Roger Campione en un reciente libro $^{7}$, nuestra realidad es aquella en la que lo silícico adquiere un protagonismo cada vez mayor.

Entendida a partir de su base carbónica, la vida ha constituido el ámbito de nuestras relaciones, singularizando la relación entre los seres vivos, o más concretamente entre los seres humanos, respecto a las relaciones entre los seres humanos y otros seres vivos y las cosas. Así entendida, la vida ha constituido un ámbito privilegiado de nuestra reflexión. Pero parece que esta comprensión de la vida como ámbito de referencia de lo humano ha perdido centralidad, ya que ahora tenemos la posibilidad (y en muchos casos la necesidad) de relacionarnos con otras realidades (máquinas, robots), en relación con las cuales no nos habíamos planteado su status ontológico. Es aquí donde sufrimos la urgencia de caracterizar a lo vivo y a lo humano. En este esfuerzo, podemos pensar en la intencionalidad, en la capacidad de autoconciencia o de recurrir al discurso simbólico. Pero también en la capacidad de reproducción.

Si la figura del faraón de la que nos habla Persio Tincani parecía un autómata porque hacía las cosas que hacen los autómatas, la pregunta está servida: ¿qué es lo que hacen los humanos y qué, por lo tanto, sirve para diferenciarlos de otras realidades ontológicas? La pregunta, como digo, es urgente, y la respuesta tiene sus riesgos, que son los que tienen que ver con el establecimiento de una especie de norma de lo humano, una especie de canon humano, que implicaría -en tanto que norma, en tanto que canon-

${ }^{7}$ Vid. Roger CAMPIONE, La plausibilidad del Derecho en la era de la inteligencia artificial. Filosofía carbónica y filosofía silícica del Derecho, Dykinson, Madrid, 2020. 
diferenciar y excluir, incluso, a lo mejor, dentro de lo que consideramos humano a priori. Lo cual afectaría, si duda, a la riqueza y multiplicidad de las manifestaciones a través de las cuales se expresa lo humano. De nuevo, la identidad y sus límites.

Persio Tincani señalaba que el libro se centra en la cuestión de la identidad y, en efecto, terminamos hablando de la identidad, en este caso de la identidad más problemática por la que nos podemos preguntar, la nuestra. Si nuestro tiempo es el tiempo en el que nos vemos obligados a plantearnos esta pregunta, podemos afirmar que, a pesar de lo que muchos puedan pensar, son buenos tiempos para la filosofía. Esta circunstancia posiblemente no distinga nuestro tiempo, pero conviene recordarla. Y si en la literatura podemos encontrar una invitación a plantearnos esta pregunta, hay que reconocer entonces que comparte con la filosofía la responsabilidad de provocar el pensamiento, la reflexión, la duda, más allá de la respuesta que podamos ofrecer a esta y a otras preguntas. Ambos, filosofía y literatura, nos maravillan en la búsqueda de nuestra identidad en un doble sentido: en lo asombroso de lo que nos podemos encontrar y en lo apasionante de la tarea que tenemos por delante. 